\title{
The effect of mechanical strains on the ferroeletric and dielectric properties of a model single crystal - Phase field simulation
}

\author{
Jie Wang, ${ }^{1}$ Yulan Li, ${ }^{2}$ Long-Qing Chen, ${ }^{2}$ and Tong-Yi Zhang ${ }^{1, *}$ \\ ${ }^{1}$ Department of Mechanical Engineering, Hong Kong University of Science and Technology, Clear \\ Water Bay, Kowloon, Hong Kong, China \\ ${ }^{2}$ Department of Materials Science and Engineering, The Pennsylvania State University, University \\ Park, Pennsylvania 16802, USA
}

\begin{abstract}
The effect of applied mechanical strains on the ferroelectric and dielectric properties of a model single crystal is investigated using a phase field model based on the time-dependent Ginzburg-Landau equation, which takes both multiple-dipole-dipole-electric and -elastic interactions into account. The evolution of the ferroelectric domain structure is simulated at different temperatures and applied strains. The results show that the paraelectric/ferroelectric phase transition temperature linearly increases with the applied mechanical strain under mechanical clamping conditions. Analogous to the classical Ehrenfest equation, a thermodynamics equation is derived to describe the relationship between the transition temperature and the applied strain. The change in the domain structure with temperature under applied inequiaxial strains is different from that under applied equiaxial strains. The simulations also illustrate the changes in the coercive field, the remanent polarization and the nonlinear dielectric constant with the applied strain.
\end{abstract}

Keywords: Phase field models, Dielectric, Simulation, Ferroelectricity, Microstructure

\footnotetext{
*Corresponding author, Tel: (852) 2358-7192, Fax: (852) 2358-1543, E-mail: mezhangt@ust.hk
} 


\section{Introduction}

Ferroelectric materials have become preferred materials in a wide variety of electronic and mechatronic devices due to their pronounced dielectric, piezoelectric, and pyroelectric properties. In applications, ferroelectric devices usually undergo external mechanical loading, such as the mismatch strain between a ferroelectric thin film and its associated substrate, hydrostatic pressure in deep-water environments, etc. Mechanical loads and/or constraints may cause lattice distortion, domain wall motion, and changes in the domain structure [1] and thus shift the paraelectric/ferroelectric phase transition temperature and vary the ferroelectric and dielectric properties. These effects induced by mechanical loads and/or constraints may enhance or damage the performance of devices made of ferroelectric materials. Therefore, it is of great importance to understand and predict the effects of applied mechanical strains on the paraelectric/ferroelectric phase transition and on the ferroelectric and dielectric properties of ferroelectric materials.

A ferroelectric material exhibits spontaneous polarization, spontaneous strain and a domain structure below its Curie temperature. Inside an electric domain, microscopic polarizations are homogeneously distributed and have the same orientation. A domain structure consists of domains, which have different orientations, and boundaries between the domains, which are called domain walls. Sufficiently high electrical and/or mechanical loads can change the polarizations and thus result in domain nucleation, domain wall motion, domain switching and/or a complete change in the domain structure. When a sufficiently high applied electric field revises its direction, the macroscopic polarization of an electric field-loaded ferroelectric material will switch $180^{\circ}$ and exhibit a hysteresis loop of polarization versus the electric field. The coercive field, the remanent polarization and the nonlinear dielectric constant are all defined based on the hysteresis loop. In 
addition to electric fields, temperature and mechanical loads and/or constraints play important roles in the material behavior of ferroelectrics. For example, spontaneous polarization decreases as temperature increases and vanishes above the Curie temperature in ferroelectric material. A high applied compressive stress is able to convert a polydomain structure into a single domain [2].

In the study of the effects of temperature, external mechanical loads and/or constraints on the phase diagram, the spontaneous polarization, the coercive field, the remanent polarization and the dielectric constant of ferroelectrics [3-14], thermodynamic theories are widely employed. For example, Oh and Jang [9] developed a two-dimensional thermodynamic model to investigate the effects of film stresses on the phase stability and various ferroelectric properties of epitaxially oriented $\mathrm{Pb}(\mathrm{Zr}, \mathrm{Ti}) \mathrm{O}_{3}$ thin films. A nonlinear thermodynamic theory was used by Emelyanov et al. [8] to describe the influence of external mechanical constraints on the ferroelectric, dielectric and piezoelectric properties of epitaxial thin films. They predicted that the stress-induced ferroelectricto-paraelectric phase transition might take place at room temperature in a $\mathrm{BaTiO}_{3}$ or $\mathrm{PbTiO}_{3}$ thin film subjected to a large compressive mismatch strain. Phase field models are based on thermodynamic theories and kinetics and take into account the gradient energy, i.e., the domain wall energy, and the multiple-dipole-dipole-electric and -elastic interaction energies, which play important roles in the formation of domain structures and in the arrangements of dipoles at the domain walls $[15,16]$. Moreover, phase field models allow us to calculate the evolution of a microstructure, thereby providing detailed information about the microstructure and the material behavior [15-24]. 
In a phase field model, thermodynamic energies are described in terms of a set of continuous order parameters. The temporal evolution of a microstructure is obtained by solving kinetic equations that govern the time-dependence of the spatially inhomogeneous order parameters. A phase field model does not make any prior assumptions about transient microstructures, which may appear on a phase transformation path. Phase transformation is a direct consequence of the minimization process of the total free energy of an entire simulated system. Phase field models have been widely used to study the domain structures in ferroelectric materials and polarization switching [15-17,25-31]. For instance, Cao and Cross [25] proposed a three-dimensional Laudau-Ginzburg model to describe the tetragonal twin microstructure in ferroelectrics. They presented quasi-onedimensional analytic solutions for the space profiles of the order parameters for $180^{\circ}$ - and $90^{\circ}$-twin microstructures. Based on the time-dependent Ginzburg-Landau equation, Nambu and Sagala [26] simulated domain structures in ferroelectrics with consideration of multiple-dipole-dipole-elastic interactions. By taking into account multiple-dipole-dipole-electric and -elastic interactions, $\mathrm{Hu}$ and Chen $[15,16]$ conducted two- and three-dimensional phase field simulations of ferroelectric domain structures and found that the multiple-dipole-dipole-elastic interactions played a critical role in the formation of the twin microstructures, whereas the multiple-dipole-dipole-electric interactions were responsible for the head-to-tail arrangements of the dipoles at the twin boundaries (domain walls). Li et al. [28,29] extended the phase field model to study the stability and evolution of domain microstructures in thin films. Ahluwalia and Cao [30,31] investigated the size dependence of domain patterns and the influence of dipolar defects on the switching behavior of ferroelectrics. Recently, Wang et al. [17] simulated ferroelectric/ferroelastic polarization switching based on a phase field model, which took into account the multiple-dipole-dipole-electric and -elastic 
interactions. Following this previous work [17], the present work further studies the effect of applied mechanical strains on a single crystal under fully clamped conditions.

The effect of applied mechanical strain on the phase transition and on the ferroelectric and dielectric properties of a fully clamped single crystal is investigated in the present work using a phase field model that is based on the time-dependent Ginzburg-Landau equation and takes the multiple-dipole-dipole-elastic and -electric interactions into account. We numerically simulate the electric field dependence of the polarization under different applied mechanical strains, which yields the nonlinear hysteresis loops of polarization versus external electric field under different applied strains. From the hysteresis loops, we have the changes in the coercive field, the remanent polarization and the nonlinear dielectric constant with the applied strains.

\section{Simulation methodology}

\subsection{General approach of the phase field model}

The paraelectric-to-ferroelectric phase transition occurs in a ferroelectric material when its temperature is lower than its Curie point. The spontaneous polarization vector, $\mathbf{P}=\left(P_{1}, P_{2}, P_{3}\right)$, is usually used as the order parameter to calculate thermodynamic energies of the ferroelectric phase in the Laudau phase transformation theory. In phase-field simulations, the time-dependent Ginzburg-Landau equation,

$$
\frac{\partial P_{i}(\mathbf{r}, t)}{\partial t}=-L \frac{\delta F}{\delta P_{i}(\mathbf{r}, t)} \quad(i=1,2,3),
$$


is generally used to calculate the temporal evolution, where $L$ is the kinetic coefficient, $F$ is the total free energy of the system, $\delta F / \delta P_{i}(\mathbf{r}, t)$ represents the thermodynamic driving force of the spatial and temporal evolution of the simulated system, $\mathbf{r}$ denotes the spatial vector, $\mathbf{r}=\left(x_{1}, x_{2}, x_{3}\right)$, and $t$ denotes time. The total free energy of the system includes the bulk free energy, the domain wall energy, i.e., the energy of the spontaneous polarization gradient, the multiple-dipole-dipole-electric and -elastic interaction energies, and the elastic energy and the electric energy induced by applied electrical and mechanical loads.

The bulk Landau free energy density is commonly expressed by a six-order polynomial of the polarization components

$$
\begin{aligned}
& f_{L}\left(P_{i}\right)=\alpha_{1}\left(P_{1}^{2}+P_{2}^{2}+P_{3}^{2}\right)+\alpha_{11}\left(P_{1}^{4}+P_{2}^{4}+P_{3}^{4}\right)+\alpha_{12}\left(P_{1}^{2} P_{2}^{2}+P_{2}^{2} P_{3}^{2}+P_{1}^{2} P_{3}^{2}\right) \\
& \quad+\alpha_{111}\left(P_{1}^{6}+P_{2}^{6}+P_{3}^{6}\right)+\alpha_{112}\left[\left(P_{1}^{4}\left(P_{2}^{2}+P_{3}^{2}\right)+P_{2}^{4}\left(P_{1}^{2}+P_{3}^{2}\right)+P_{3}^{4}\left(P_{1}^{2}+P_{2}^{2}\right)\right]\right. \\
& \quad+\alpha_{123} P_{1}^{2} P_{2}^{2} P_{3}^{2},
\end{aligned}
$$

where $\alpha_{11}, \alpha_{12}, \alpha_{111}, \alpha_{112}, \alpha_{123}$ are constant coefficients and $\alpha_{1}=\left(T-T_{0}\right) / 2 \varepsilon_{0} C_{0} . T$ and $T_{0}$ denote the temperature and the Curie-Weiss temperature, respectively. $C_{0}$ is the Curie constant and $\varepsilon_{0}$ is the dielectric constant of vacuum.

In the Ginzburg-Landau theory, the free energy function also depends on the gradient of the order parameter. For ferroelectric materials, the polarization gradient energy represents the domain wall energy. For simplicity, the lowest order of the gradient energy density is used here, which takes the form: 


$$
\begin{aligned}
f_{G}\left(P_{i, j}\right)= & \frac{1}{2} G_{11}\left(P_{1,1}^{2}+P_{2,2}^{2}+P_{3,3}^{2}\right)+G_{12}\left(P_{1,1} P_{2,2}+P_{2,2} P_{3,3}+P_{1,1} P_{3,3}\right) \\
& +\frac{1}{2} G_{44}\left[\left(P_{1,2}+P_{2,1}\right)^{2}+\left(P_{2,3}+P_{3,2}\right)^{2}+\left(P_{1,3}+P_{3,1}\right)^{2}\right] \\
& +\frac{1}{2} G_{44}^{\prime}\left[\left(P_{1,2}-P_{2,1}\right)^{2}+\left(P_{2,3}-P_{3,2}\right)^{2}+\left(P_{1,3}-P_{3,1}\right)^{2}\right],
\end{aligned}
$$

where $G_{11}, G_{12}, G_{44}$, and $G_{44}^{\prime}$ are gradient energy coefficients, and $P_{i, j}$ denotes the derivative of the $i$ th component of the polarization vector, $P_{i}$, with respect to the $j$ th coordinate and $i, j=1,2,3$.

The elastic energy density, including the multiple-dipole-dipole-elastic interaction, is calculated from

$$
f_{\text {ela }}=\frac{1}{2} c_{i j k l} \varepsilon_{i j}^{e l a} \varepsilon_{k l}^{e l a}
$$

where $c_{i j k l}$ are the elastic constants, and $\varepsilon_{i j}^{e l a}$ are the elastic strain and take, if there is no applied strain, the form of $\varepsilon_{i j}^{e l a}=\varepsilon_{i j}^{(s)}-\varepsilon_{i j}^{0}$, in which $\varepsilon_{i j}^{(s)}$ and $\varepsilon_{i j}^{0}$ are the local strain and eigenstrain, respectively. The local strains, $\varepsilon_{i j}^{(s)}$, related to the polarization, are calculated based on the general eigenstrain theory described as follows. The eigenstrains are linked to the polarization components in the following form, 


$$
\begin{aligned}
& \varepsilon_{11}^{0}=Q_{11} P_{1}^{2}+Q_{12}\left(P_{2}^{2}+P_{3}^{2}\right), \\
& \varepsilon_{22}^{0}=Q_{11} P_{2}^{2}+Q_{12}\left(P_{3}^{2}+P_{1}^{2}\right), \\
& \varepsilon_{33}^{0}=Q_{11} P_{3}^{2}+Q_{12}\left(P_{1}^{2}+P_{2}^{2}\right), \\
& \varepsilon_{23}^{0}=Q_{44} P_{2} P_{3}, \\
& \varepsilon_{13}^{0}=Q_{44} P_{1} P_{3}, \\
& \varepsilon_{12}^{0}=Q_{44} P_{1} P_{2},
\end{aligned}
$$

where $Q_{11}, Q_{12}$ and $Q_{44}$ are the electrostrictive coefficients according to Voigt's notation. When a dipole is among other dipoles with different orientations, there exist long-range dipole-dipoleelastic interactions. The long-range elastic interactions play an essential role in the formation of a domain structure. A twin-like domain structure is formed when multiple-dipole-dipole-elastic interactions predominate [26]. It is still a challenging task to calculate the long-range elastic interaction energy for arbitrary boundary conditions analytically. However, with periodic boundary conditions, which are adopted in this study, the general solution of the elastic displacement field is given in Fourier space by [32]

$$
\bar{u}_{i}^{(s)}(\xi)=X_{j} N_{i j}(\xi) / D(\xi),
$$

where $X_{i}=-I c_{i j k l} \varepsilon_{k l}^{0} \xi_{j}, I=\sqrt{-1}, N_{i j}(\xi)$ are cofactors of a $3 \times 3$ matrix $\mathbf{K}(\xi)$,

$$
\mathbf{K}(\xi)=\left[\begin{array}{lll}
K_{11} & K_{12} & K_{13} \\
K_{21} & K_{22} & K_{23} \\
K_{31} & K_{32} & K_{33}
\end{array}\right],
$$

and $D(\xi)$ is the determinant of matrix $\mathbf{K}(\xi)$. Note that $K_{k i}(\xi)=c_{k j i l} \xi_{j} \xi_{l}$, in which $c_{i j k l}$ and $\xi_{i}$ are the elastic constant components and coordinates in Fourier space, respectively. 
For ferroelectric materials with the perovskite crystal structure, spontaneous polarization changes the crystal system from cubic to tetragonal. In the present simulations, we use the cubic paraelectric phase as the background material such that all electric dipoles are embedded within the background material. Below the Curie temperature the elastic properties of a ferroelectric tetragonal crystal are not cubic and are inhomogeneous for a multi-domain structure. For simplicity, the cubic and the homogeneous approximations for the elastic properties are made to obtain the elastic field as in the previous works [26-31]. For cubic crystals, the explicit expressions of $D(\xi)$ and $N_{i j}(\xi)$ are

$$
\begin{gathered}
D(\xi)=\mu^{2}\left(\lambda+2 \mu+\mu^{\prime}\right) \xi^{6}+\mu^{\prime}\left(2 \lambda+2 \mu+\mu^{\prime}\right) \xi^{2}\left(\xi_{1}^{2} \xi_{2}^{2}+\xi_{2}^{2} \xi_{3}^{2}+\xi_{1}^{2} \xi_{3}^{2}\right) \\
+\mu^{\prime 2}\left(3 \lambda+3 \mu+\mu^{\prime}\right) \xi_{1}^{2} \xi_{2}^{2} \xi_{3}^{2}, \\
N_{11}(\xi)=\mu^{2} \xi^{4}+\beta \xi^{2}\left(\xi_{2}^{2}+\xi_{3}^{2}\right)+\gamma \xi_{2}^{2} \xi_{3}^{2}, \\
N_{12}=-(\lambda+\mu) \xi_{1} \xi_{2}\left(\mu \xi^{2}+\mu^{\prime} \xi_{3}^{2}\right),
\end{gathered}
$$

and the other components are obtained by the cyclical permutation of $1,2,3$, where

$$
\begin{array}{r}
\xi^{2}=\xi_{i} \xi_{i}, \beta=\mu\left(\lambda+\mu+\mu^{\prime}\right), \gamma=\mu^{\prime}\left(2 \lambda+2 \mu+\mu^{\prime}\right), \\
\lambda=c_{12}, \mu=c_{44}, \text { and } \mu^{\prime}=c_{11}-c_{12}-2 c_{44},
\end{array}
$$

in which, $c_{11}, c_{12}$ and $c_{44}$ are three independent elastic constants according to Voigt's notation.

Spontaneous polarization changes the dimensions and/or orientations of each crystalline cell in a ferroelectric and thus produces elastic displacements, $u_{i}^{(s)}, \mathrm{i}=1,2,3$, and hence local strains, $\varepsilon_{i j}^{(s)}$ :

$$
\varepsilon_{i j}^{(s)}=\frac{1}{2}\left(u_{i, j}^{(s)}+u_{j, i}^{(s)}\right)
$$

When there exists an externally applied homogeneous strain, $\varepsilon_{i j}^{(a)}$, the elastic strains, $\varepsilon_{i j}^{e l a}$, in Eq. (4) should be: 


$$
\varepsilon_{i j}^{e l a}=\varepsilon_{i j}^{(s)}-\varepsilon_{i j}^{0}+\varepsilon_{i j}^{(a)} .
$$

As described above, the elastic strain energy density, $f_{e l a}\left(P_{i}, \varepsilon_{i j}^{(a)}\right)$, is a function of polarization and applied strains.

In the present phase-field simulations, each element is represented by an electric dipole with the strength of the dipole given by a local polarization vector. The electric field produced by all dipoles forms an internal electric field, which, in turn, exerts a force on each of the dipoles. The multipledipole-dipole-electric interactions play a crucial role in the formation of a head-to-tail domain structure and in the process of domain switching as well [16]. The multiple-dipole-dipole-electric interaction energy density is calculated from

$$
f_{d i p}=-\frac{1}{2} E_{i}^{d i p} P_{i}
$$

where $E_{i}^{d i p}$ is the $i$ th component of the electric field,

$$
\mathbf{E}^{d i p}(\mathbf{r})=-\frac{1}{4 \pi \varepsilon} \int\left\{\frac{\mathbf{P}\left(\mathbf{r}^{\prime}\right)}{\left|\mathbf{r}-\mathbf{r}^{\prime}\right|^{3}}-\frac{3\left[\left(\mathbf{r}-\mathbf{r}^{\prime}\right)\right]\left[\mathbf{P}\left(\mathbf{r}^{\prime}\right) \cdot\left(\mathbf{r}-\mathbf{r}^{\prime}\right)\right]}{\left|\mathbf{r}-\mathbf{r}^{\prime}\right|^{5}}\right\} d^{3} r^{\prime}
$$

at point $\mathbf{r}$, induced by all dipoles, in which $\varepsilon^{\prime}$ is a linear dielectric constant, which will be described in detail in the following. When there is an externally applied electric field, $E_{i}^{(a)}$, an additional electrical energy density,

$$
f_{\text {elec }}=-E_{i}^{(a)} P_{i}
$$

should be taken into account in the simulations. 
Integrating the free energy density over the entire volume of a simulated ferroelectric material yields the total free energy, $F$, of the simulated material under externally applied strains, $\varepsilon_{i j}{ }^{(a)}$, and an externally applied electric field, $E_{i}^{(a)}$. Mathematically, we have,

$$
F=\int_{V}\left[f_{L}\left(P_{i}\right)+f_{G}\left(P_{i, j}\right)+f_{\text {dip }}\left(P_{i}\right)+f_{\text {elec }}\left(P_{i}, E_{i}^{(a)}\right)+f_{\text {ela }}\left(P_{i}, \varepsilon_{i j}^{(a)}\right)\right] d V,
$$

where $V$ is the volume of the simulated ferroelectric material. By substituting Eq. (15) into Eq. (1), the temporal evolution of the domain structures at different temperatures and applied strains and the response of polarization to an applied electric field under different applied strains can be obtained after solving the equation numerically.

For convenience, we employ the following set of the dimensionless variables for Eq. (1) [16,17].

$$
\begin{gathered}
\mathbf{r}^{*}=\sqrt{\left|\alpha_{0}\right| / G_{110}} \mathbf{r}, t^{*}=\left|\alpha_{0}\right| L t, \mathbf{P}^{*}=\mathbf{P} / P_{0}, E_{i}^{(a),{ }^{*}}=E_{i}^{(a)} /\left(\left|\alpha_{0}\right| P_{0}\right), \\
\alpha_{1}^{*}=\alpha_{1} /\left|\alpha_{0}\right|, \alpha_{11}^{*}=\alpha_{11} P_{0}^{2} /\left|\alpha_{0}\right|, \alpha_{12}^{*}=\alpha_{12} P_{0}^{2} /\left|\alpha_{0}\right|, \\
\alpha_{111}^{*}=\alpha_{11} P_{0}^{4} /\left|\alpha_{0}\right|, \alpha_{112}^{*}=\alpha_{112} P_{0}^{4} /\left|\alpha_{0}\right|, \alpha_{123}^{*}=\alpha_{123} P_{0}^{4} /\left|\alpha_{0}\right|, \\
Q_{11}^{*}=Q_{11} P_{0}^{2}, Q_{12}^{*}=Q_{12} P_{0}^{2}, Q_{44}^{*}=Q_{44} P_{0}^{2}, \\
c_{11}^{*}=c_{11} /\left(\left|\alpha_{0}\right| P_{0}^{2}\right), c_{12}^{*}=c_{12} /\left(\left|\alpha_{0}\right| P_{0}^{2}\right), c_{44}^{*}=c_{44} /\left(\left|\alpha_{0}\right| P_{0}^{2}\right), \\
G_{11}^{*}=G_{11} / G_{110}, G_{12}^{*}=G_{12} / G_{110}, G_{44}^{*}=G_{44} / G_{110}, G_{44}^{* *}=G_{44}^{\prime} / G_{110},
\end{gathered}
$$

where $P_{0}$ is the magnitude of the spontaneous polarization at room temperature, $G_{110}$ is a reference value of the gradient energy coefficients and $\alpha_{0}$ represents the value of $\alpha_{1}$ at $25^{\circ} \mathrm{C}$. The superscript asterisk, ${ }^{*}$, denotes the dimensionless value of the corresponding variable. 
With the dimensionless variables and Eq. (15), we rewrite the time-dependent Ginzburg-Landau equation as

$$
\frac{\partial P_{i}^{*}\left(\mathbf{r}^{*}, t^{*}\right)}{\partial t^{*}}=-\frac{\delta \int\left[f_{L}\left(P_{i}^{*}\right)+f_{\text {dip }}\left(P_{i}^{*}\right)+f_{\text {elec }}\left(P_{i}^{*}, E_{i}^{(a), *}\right)+f_{\text {ela }}\left(P_{i}^{*}, \varepsilon_{i j}^{(a)}\right)\right] d V^{*}}{\delta P_{i}^{*}}-\frac{\delta \int f_{G}\left(P_{i . j}^{*}\right) d V^{*}}{\delta P_{i}^{*}} .
$$

In Fourier space, Eq. (17) takes the form

$$
\frac{\partial}{\partial t^{*}} \hat{P}_{i}\left(\xi, t^{*}\right)=-\left\{\hat{f}\left(P_{i}^{*}\right)\right\}_{\xi}-G_{i} \hat{P}_{i}\left(\xi, t^{*}\right)
$$

where $\hat{P}_{i}\left(\xi, t^{*}\right)$ and $\left\{\hat{f}\left(P_{i}^{*}\right)\right\}_{\xi}$ are the Fourier transformations of $P_{i}^{*}\left(\mathbf{r}^{*}, t^{*}\right)$ and $\frac{\delta \int\left[f_{L}\left(P_{i}^{*}\right)+f_{\text {dip }}\left(P_{i}^{*}\right)+f_{\text {elec }}\left(P_{i}^{*}, E_{i}^{(a),,^{*}}\right)+f_{\text {ela }}\left(P_{i}^{*}, \varepsilon_{i j}^{(a)}\right)\right] d V^{*}}{\delta P_{i}^{*}}$, respectively. The $G_{i} \mathrm{~s}$ are the gradient operators corresponding to the $i$ th-component of the polarization field and are defined as follows:

$$
\begin{aligned}
& G_{1}=G_{11}^{*} \xi_{1}^{2}+\left(G_{44}^{*}+G_{44}^{* *}\right)\left(\xi_{2}^{2}+\xi_{3}^{2}\right), \\
& G_{2}=G_{11}^{*} \xi_{2}^{2}+\left(G_{44}^{*}+G_{44}^{* *}\right)\left(\xi_{1}^{2}+\xi_{3}^{2}\right), \\
& G_{3}=G_{11}^{*} \xi_{3}^{2}+\left(G_{44}^{*}+G_{44}^{* *}\right)\left(\xi_{2}^{2}+\xi_{1}^{2}\right) .
\end{aligned}
$$

The semi-implicit Fourier-spectral method [16] was employed to solve the partial differential equation (18).

\section{2. $2 D$ simulation and used parameters}

Two-dimensional (2D) simulations are conducted under the plane-strain condition with all the strains related to the $x_{3}$ axis being zero, although the methodology described in Section 2.1 can be applied to three-dimensional simulations as well. In the present 2D simulation, the polarization component, $P_{3}$, is treated as zero. The dielectric constant, $\varepsilon^{\prime}$, introduced in Eq. (13) is usually 
related to the coefficient of the first right-hand term in Eq. (2), i.e., the second order of polarizations. As mentioned above, $\alpha_{1}=\left(T-T_{0}\right) / 2 \varepsilon_{0} C_{0}$ is a function of temperature under the constant stress condition. Under the constant strain condition, the coefficient of the second order of the polarization should be modified to $\alpha_{1}^{e}=\alpha_{1}-\left(q_{11} \varepsilon_{11}^{(a)}+q_{12} \varepsilon_{22}^{(a)}\right)$ for $P_{1}$ and to $\alpha_{2}^{e}=\alpha_{1}-\left(q_{11} \varepsilon_{22}^{(a)}+q_{12} \varepsilon_{11}^{(a)}\right)$ for $P_{2}$ in a $2 \mathrm{D}$ system (see the Appendix for details). In the present model, we use $\varepsilon^{\prime}=1 /\left(2 \alpha_{1}^{e}\right)$ for $E_{1}^{d i p}$ and $\varepsilon^{\prime}=1 /\left(2 \alpha_{2}^{e}\right)$ for $E_{2}^{d i p}$ when $\alpha_{1}^{e}$ and $\alpha_{2}^{e}$ are larger than zero, respectively, and $\varepsilon^{\prime}=-1 /\left(4 \alpha_{1}^{e}\right)$ for $E_{1}^{d i p}$ and $\varepsilon^{\prime}=-1 /\left(4 \alpha_{2}^{e}\right)$ for $E_{2}^{d i p}$ when $\alpha_{1}^{e}$ and $\alpha_{2}^{e}$ are less than zero, respectively. Clearly, the dielectric constant, $\varepsilon^{\prime}$, is a function of the temperature and the applied strains.

Here, we set the magnitude of the spontaneous polarization at room temperature to $P_{0}=\left|\mathbf{P}_{0}\right|=0.757 \mathrm{C} / \mathrm{m}^{2}$, the reference value of the gradient energy coefficients to $G_{110}=1.73 \times 10^{-10} \mathrm{~m}^{4} \mathrm{~N} / \mathrm{C}^{2}$ and the value of $\alpha_{1}$ at $25^{\circ} \mathrm{C}$ to $\alpha_{0}=\alpha_{1,25^{\circ} \mathrm{C}}=\left(T-T_{0}\right) /\left(2 \varepsilon_{0} C_{0}\right)=(25-479) \times 3.8 \times 10^{5} \mathrm{~m}^{2} \mathrm{~N} / \mathrm{C}^{2}$, where $T$ is the temperature with a unit of ${ }^{\circ} \mathrm{C}[28]$. The values of the dimensionless (normalized) material coefficients of $\mathrm{PbTiO}_{3}$ used in the simulations are listed in Table I.

Table I. Values of the normalized coefficients used in the simulation

\begin{tabular}{ccccccccccccccc}
\hline$\alpha_{11}^{*}$ & $\alpha_{12}^{*}$ & $\alpha_{111}^{*}$ & $\alpha_{112}^{*}$ & $\alpha_{123}^{*}$ & $Q_{11}^{*}$ & $Q_{12}^{*}$ & $Q_{44}^{*}$ & $c_{11}^{*}$ & $c_{12}^{*}$ & $c_{44}^{*}$ & $G_{11}^{*}$ & $G_{12}^{*}$ & $G_{44}^{*}$ & $G_{44}^{* *}$ \\
\hline-0.24 & 2.5 & 0.49 & 1.2 & -7.0 & 0.05 & -0.015 & 0.019 & 1766 & 802 & 1124 & 1.60 & 0.0 & 0.8 & 0.8 \\
\hline
\end{tabular}


In the simulations, we use $32 \times 32$ discrete grid points with a cell size of $\Delta x_{1}^{*}=\Delta x_{2}^{*}=1$ and adopt periodic boundary conditions in both the $x_{1}$ - and $x_{2}$-directions. The periodic boundary conditions imply that the simulated domain structure denotes the one periodic domain structure of a model single crystal in which the domains are distributed periodically. The domain structure is represented by the polarization field, which varies spatially and, at each lattice site, is characterized by a twocomponent vector in the $2 \mathrm{D}$ simulations. The length and direction of the vector denote the magnitude and direction of the polarization, respectively. The time step is set to $\Delta t^{*}=0.04$ and total number of steps is 2000 in solving Eq. (18), which can ensure that the domain structure reaches its steady state [17]. We report the simulation results only at the steady state.

\section{Simulation results and discussion}

\subsection{The effects of external applied strain on the ferroelectric properties}

An equiaxial strain, $\varepsilon_{11}^{(a)}=\varepsilon_{22}^{(a)}=\varepsilon^{(a)}$ with $\varepsilon_{12}^{(a)}=0$, was applied to the simulated system prior to simulations. Figures (1a), (1b), (1c) and (1d) show the ferroelectric domain structures at room temperature under different strains of $\varepsilon^{(a)}=0.008,0,-0.008$ and -0.012 , respectively. Under $\varepsilon^{(a)}=0.008$, the domains form a twin structure with $90^{\circ}$ domain walls between two adjacent domains. When the strain decreases from 0.008 to 0 , to -0.008 , and to -0.012 , the magnitudes of the spontaneous polarizations become smaller and smaller, as shown in Figs. (1a), (1b), (1c) and (1d).

Further decreasing the strain reduces the magnitude of the spontaneous polarization eventually to zero and thus the domain structure disappears. 
Under the sustained strain boundary condition, the change in the spontaneous polarization causes a change in the internal stresses. The internal stresses related to the spontaneous polarization were calculated from Hooke's law as $\sigma_{i j}^{*}=c_{i j k l}^{*}\left(\varepsilon_{k l}^{(s)}-\varepsilon_{i j}^{0}\right)$. Figs. (1A), (1B), (1C) and (1D) show the stress distributions of $\sigma_{11}^{*}+\sigma_{22}^{*}$ corresponding to the domain structures shown in Figs. (1a), (1b), (1c) and (1d), respectively. Clearly, the internal stress field is inhomogeneous due to the inhomogeneous distributions of the spontaneous polarizations. The results indicate that a tensile sustained strain enhances the magnitude of the internal stresses, whereas a compressive sustained strain reduces it. This is because a tensile (or compressive) sustained strain increases (or decreases) the magnitude of the spontaneous polarization.

Figure 2 shows the temperature dependence of the average spontaneous polarization for different applied strains. The average spontaneous polarization, $\left\langle P^{*}{ }_{s}\right\rangle$, is obtained by averaging the polarization magnitude, $P_{\mathrm{s}}^{*}=\sqrt{P_{1}^{* 2}+P_{2}^{* 2}}$, at each grid, over the entire simulated ferroelectric material. Under a sustained strain, the average spontaneous polarization decreases continuously down to zero with an increase of temperature. At a given temperature, a tensile strain increases the average spontaneous polarization, while a compressive strain reduces it. A continuous change of the average spontaneous polarization with temperature implies that the paraelectric/ferroelectric phase transition of $\mathrm{PbTiO}_{3}$ crystals is of the second order under a sustained strain. Under a sustained stress, however, the paraelectric/ferroelectric phase transition is of the first order [13]. Applying a sustained strain linearly changes the phase transition temperature, i.e., the Curie temperature, $T_{c}$. The linear change is described by $T_{c}=480+31250 \times \varepsilon^{(a)}$ and is shown in the inset of Fig. 2, indicating that a tensile strain linearly increases the Curie temperature, while a compressive strain linearly reduces it. This result is different from the results from the effect of a misfit strain on the transition 
temperature in ferroelectric thin films $[3,6,19]$, in which both the compressive strain and the tensile strain linearly increase the Curie temperature. This is because the plane strain condition is adopted and $P_{3}$ is treated as zero here, while the plane stress condition, $\sigma_{33}=\sigma_{13}=\sigma_{23}=0$, is used and $P_{3}$ is treated as nonzero in the ferroelectric thin films $[3,6,19]$.

The average internal stress, $\left\langle\sigma_{i j}^{*}\right\rangle$, induced by the spontaneous polarization was calculated by averaging the internal stress, $\sigma_{i j}^{*}$, at each grid over the entire simulated material. Under a sustained biaxial strain, $\varepsilon_{11}^{(a)}=\varepsilon_{22}^{(a)}=\varepsilon^{(a)}$, the average internal stresses in the $x_{1}$ and $x_{2}$ directions are equal, i.e., $\left\langle\sigma_{11}^{*}\right\rangle=\left\langle\sigma_{22}^{*}\right\rangle=\left\langle\sigma^{*}\right\rangle$. Figure 3 illustrates the temperature dependence of the average internal stress, $\left\langle\sigma^{*}\right\rangle$, under different sustained strains, showing that the average internal stresses are all compressive. This is because only the spontaneous polarization-induced internal stress is considered and the Curie temperature varies with the sustained strain. For a given sustained strain, the magnitude of the average internal stress linearly increases as the temperature decreases. The slopes of the curves are almost the same, as shown in Fig. 3. Fig. 4 shows the strain dependence of the average internal stress $\left\langle\sigma^{*}\right\rangle$ at a constant temperature, indicating that there is a linear relationship between the average internal stress and the sustained strain and that the average internal stress versus the sustained strain for different temperatures has the same slope.

In a paraelectric/ferroelectric phase transition under a sustained strain, the average internal stress, which is related to the spontaneous polarization, is a function of temperature and the sustained strain in the absence of external electric fields. Thus, we can write the average internal stress, $\left\langle\sigma^{*}\right\rangle$, in a differentiated form: 


$$
d<\sigma^{*}>=\left(\frac{\partial<\sigma^{*}>}{\partial T}\right)_{\varepsilon^{(a)}} d T+\left(\frac{\partial<\sigma^{*}>}{\partial \varepsilon^{(a)}}\right)_{T} d \varepsilon^{(a)}=\beta d T+\gamma d \varepsilon^{(a)}
$$

where $\beta \equiv\left(\frac{\partial<\sigma^{*}>}{\partial T}\right)_{\varepsilon^{(a)}}$ denotes the change rate of the average internal stress with temperature under a sustained strain and $\gamma \equiv\left(\frac{\partial<\sigma^{*}>}{\partial \varepsilon^{(a)}}\right)_{T}$ stands for the change rate of the average internal stress with the sustained strain at a given temperature. Figures (3) and (4) show that the average internal stress changes smoothly to zero in the ferroelectric phase. Note that there is no internal stress related to the spontaneous polarization in the paraelectric phase because there is no spontaneous polarization. Therefore, the average internal stress is of continuity at the phase transition point, from which we have $\left.d<\sigma^{*}\right\rangle^{\text {Ferro }}=d<\sigma^{*}>^{\text {Para }}$. Then, from Eq. (20) we have

$$
\frac{d T_{c}}{d \varepsilon^{(a)}}=-\frac{\Delta \gamma}{\Delta \beta}
$$

where $\Delta \beta=\beta^{\text {ferro }}-\beta^{\text {para }}$ and $\Delta \gamma=\gamma^{\text {ferro }}-\gamma^{\text {para }}$ are the discontinuous changes of $\beta$ and $\gamma$ at the Curie point, respectively. Noting that the average internal stress, $\left\langle\sigma^{*}\right\rangle$, is zero in the paraelectric phase, we have $\beta^{\text {para }}=0$ and $\gamma^{\text {para }}=0$ and then $\Delta \beta=\beta^{\text {ferro }}$ and $\Delta \gamma=\gamma^{\text {ferro }}$. From the simulation results shown Figs. 3 and 4 , we determine the average values of $\Delta \beta=\beta^{\text {ferro }}$ and $\Delta \gamma=\gamma^{\text {ferro }}$ to be 0.1075 and -3315 , respectively, for the ferroelectric phase. Substituting the average values into Eq. (21) leads to $\frac{d T_{c}}{d \varepsilon^{(a)}}=-\frac{-3315}{0.1075}=30837$ or $T_{c}\left(\varepsilon^{(a)}\right)=T_{c}\left(\varepsilon^{(a)}=0\right)+30837 \varepsilon^{(a)}$, which is consistent with the equation obtained in Fig. 2. For the second order ferroelectric phase transition under constant pressure, the shift in the transition temperature, $T_{\mathrm{c}}$, with the hydrostatic pressure, $p$, is described by the well-known Ehrenfest equation, $\frac{d T_{c}}{d p}=\frac{\Delta s_{v}}{\Delta \alpha_{v}}$, in which $\Delta \alpha_{v}$ and $\Delta s_{v}$ are the discontinuous changes in the volume expansions and in the compression coefficients at the Curie 
point, respectively [33]. Clearly, Equation (21) is a modified version of the Ehrenfest equation, which describes the shift of the transition temperature with the sustained strain in a fully clamped ferroelectric material.

Figure 5 shows the temperature dependence of the average spontaneous polarization under different inequiaxial strains. Under the inequiaxial strain of $\varepsilon_{11}{ }^{(a)}=0.004$ and $\varepsilon_{22}{ }^{(a)}=0$ or $\varepsilon_{11}{ }^{(a)}=-0.004$ and $\varepsilon_{22}{ }^{(a)}=0$, there is a jump in the average spontaneous polarization from point $B_{1}$ to point $C_{1}$ or from point $B_{2}$ to point $C_{2}$, as shown in Fig. 5 . The changes in the domain microstructure with temperature corresponding to $A_{1}-B_{1}-C_{1}-D_{1}$ and $A_{2}-B_{2}-C_{2}-D_{2}$ in Fig. 5 are illustrated in Fig. 6 and Fig. 7, respectively. Along with the jumps in the average spontaneous polarization in Fig. 5, the simulated ferroelectric material changes its microstructure from a multidomain state to a single-domain state, which is clearly shown in Fig. $6 \mathrm{~B}_{1}$ and Fig. $6 \mathrm{C}_{1}$ and in Fig. $7 \mathrm{~B}_{2}$ and Fig. $7 \mathrm{C}_{2}$. For comparison, Fig. 8 shows the domain structures at various temperatures under the equiaxial strain of $\varepsilon_{11}^{(a)}=\varepsilon_{22}{ }^{(a)}=-0.004$, corresponding to points $\mathrm{A}_{3}-\mathrm{B}_{3}-\mathrm{C}_{3}-\mathrm{D}_{3}$ in Fig. 5. Under the equiaxial strain, the simulated ferroelectric material maintains its multi-domain structure until the spontaneous polarization vanishes, showing completely different behavior from that under the inequiaxial strains.

\subsection{The effects of external applied strain on the nonlinear dielectric properties}

To characterize the nonlinear behaviors of ferroelectric materials under different levels of applied strains, we simulated the polarization response to different levels of external electric loading under different levels of applied equiaxial strains of $\varepsilon_{11}^{(a)}=\varepsilon_{22}^{(a)}=\varepsilon^{(a)}$. The external electric field was applied along the $x_{2}$ axis, where a positive or negative electric field meant that the field was 
parallel or anti-parallel to the $x_{2}$ direction. At each level of electric loading, we calculated the evolution of the domain structure based on Eq. (18). The volume averages of the polarizations along the $x_{2}$ direction at the steady state were taken as the macroscopic response of the simulated ferroelectric material to the applied electric field. In the simulations, the applied dimensionless field was increased from zero to a designed positive value, then, decreased from the positive value to zero and then its direction was reversed and further decreased to a designed negative value. After that, the applied electric field was increased from the designed negative value to the designed positive value. One cycle of the electric loading gives the P-E loop of the average polarization, $\left\langle P_{2}^{*}\right\rangle$, versus the electric field, $E_{2}^{(a),{ }^{*}}$. The P-E loops at room temperature were calculated under eight different levels of applied equiaxial strain. For example, Fig. 9 shows four P-E loops under the applied strains of $\varepsilon^{(a)}=-0.02, \varepsilon^{(a)}=-0.01, \varepsilon^{(a)}=-0.005$ and $\varepsilon^{(a)}=0.01$. Obviously, the hysteresis loop of the average polarization versus the electric field becomes smaller when the external applied strain, $\varepsilon^{(a)}$, changes from 0.01 to -0.01 , and vanishes when $\varepsilon^{(a)}=-0.02$. The curve with the applied strain, $\varepsilon^{(a)}=-0.02$, in Fig.9 indicates that the average polarization varies nonlinearly with the external electric field without any hysteresis loop. The vanishing of the hysteresis loop implies that the simulated ferroelectric material loses its ferroelectric property and is in the paraelectric phase, in which the polarization has the same direction as the external field. The polarization distributions verify this conclusion. Figs.10 $\left(A_{p}\right),\left(B_{p}\right),\left(C_{p}\right),\left(D_{p}\right)$ and $\left(E_{p}\right)$ show the polarization distributions corresponding to points $A_{p}, B_{p}, C_{p}, D_{p}$ and $E_{p}$ in Fig. 9, respectively. If there is no applied electric field, i.e., at point $C_{p}$, there is no spontaneous polarization in the simulated material. In the paraelectric phase, polarizations are induced by an applied electric field and the magnitude of the polarizations increases nonlinearly with the level of the applied field, as shown in Fig. 9. The polarization distribution in the ferroelectric phase is totally different from that 
in the paraelectric phase. Figs. $11\left(\mathrm{~A}_{0}\right),\left(\mathrm{B}_{0}\right),\left(\mathrm{C}_{0}\right),\left(\mathrm{D}_{0}\right),\left(\mathrm{E}_{0}\right)$ and $\left(\mathrm{F}_{0}\right)$ show the distributions of the polarizations at different levels of electric fields corresponding to points $\mathrm{A}_{0}, \mathrm{~B}_{0}, \mathrm{C}_{0}, \mathrm{D}_{0}, \mathrm{E}_{0}$ and $\mathrm{F}_{0}$ in Fig. 9 with an applied equiaxial strain of $\varepsilon^{(a)}=0.01$. During the decrease in the applied electric field from 0.8 to -0.5 , i.e., from point $\mathrm{A}_{0}$ to point $\mathrm{B}_{0}$, as shown in Fig. 9 , the average polarization decreases smoothly, which is attributed to the domain wall motion, as indicated by the domain structures illustrated in Figs. 11( $\left.\mathrm{A}_{0}\right)$ and 11( $\left.\mathrm{B}_{0}\right)$. A further decrease of the applied electric field from -0.5 to -.07 , i.e., from point $\mathrm{B}_{0}$ to point $\mathrm{C}_{0}$, cause the average polarization to decrease sharply, as shown in Fig. 9, which is attributed to the nucleation of a new domain with a different polarization direction from the parent domains, as illustrated at the lower-left corner of Fig. $11\left(\mathrm{C}_{0}\right)$. When the electric field reaches the coercive field, the average polarization jumps from positive to negative, as indicated by points $\mathrm{C}_{0}$ and $\mathrm{D}_{0}$ in Figs. 9. Figures $11\left(\mathrm{C}_{0}\right)$ and $11\left(\mathrm{D}_{0}\right)$ illustrate the domain structures before and after the jumps, thereby indicating that the domain structure changes its configuration completely. When the applied electric field increases from -0.8 to 0.5 , to 0.7 and then to 0.8 , i.e., from point $D_{0}$ to point $E_{0}$ to point $F_{0}$ and to point $A_{0}$ in Fig. 9, the average polarization increases smoothly by domain wall motion first and then increases abruptly by the nucleation of new domains and finally switches its direction completely by the entire change in the domain structure, as illustrated in Figs. 11( $\left.\mathrm{D}_{0}\right),\left(\mathrm{E}_{0}\right),\left(\mathrm{F}_{0}\right)$ and $\left(\mathrm{A}_{0}\right)$. From the hysteresis loops, we determine the coercive field, $E_{c}^{*}$, at which the average polarization reverses, and the remanent polarization, $P_{r}^{*}$, i.e., the average polarization, $\left\langle P_{2}^{*}\right\rangle$, when the applied electric field is zero. Figure 12 shows the curves of the coercive field and the remanent polarization versus the applied strain at a room temperature of $25^{\circ} \mathrm{C}$. At the applied strain of $\varepsilon^{(a)}=0.01$, the coercive field and remanent polarization are 0.75 and 0.547 , respectively. Both the coercive field and the remanent polarization decrease as the applied strain, $\varepsilon^{(a)}$ is reduced. When the applied strain decreases to -0.015 , both the coercive field and the 
remanent polarization are zero. Within the range from $\varepsilon^{(a)}=-0.015$ to $\varepsilon^{(a)}=0.01$, the variation in the coercive field with the applied strain is approximately linear, whereas the change in the remannent polarization is obviously nonlinear with the applied strain. The remanent polarization changes greatly as the applied strain varies from -0.013 to -0.015 . The strain dependence of the coercive field and the remanent polarization in Fig. 12 is very similar to the temperature dependence of the coercive field and the remanent polarization [35], indicating again that applied strains may have the same ability as temperature to vary and/or control the behavior of ferroelectric materials.

In general, there are two kinds of dielectric constants, linear and nonlinear dielectric constants, for ferroelectric materials [35]. The linear or reversible dielectric constant is defined by the slope of the P-E curve at the origin under low levels of applied electric fields such that no hysteresis loop is induced. The nonlinear or irreversible dielectric constant is defined as the slope of the P-E loop at the point with the remanent polarization. In the present work, we studied the effect of applied equiaxial strain on the nonlinear dielectric constant. From the P-E loops under various levels of applied equiaxial strains, we calculated the nonlinear dielectric constants at room temperature and plotted them in Figure 13 as a function of the applied strain. The nonlinear dielectric constant increases from 0.17 to 3.84 when the strain decreases from 0.01 to -0.015 and then decreases with the strain after -0.015 . There is a peak in the curve at $\varepsilon^{(a)}=-0.015$, at which the coercive field and the remanent polarization both decrease from finite values to zero, as indicated in Fig. 12. The vanishing of the coercive field and the remanent polarization implies that the simulated material loses its ferroelectric property, i.e., the ferroelectric-paraelectric phase transition occurs at that level 
of the applied strain, meaning that the simulated $\mathrm{PbTiO}_{3}$ is in the paraelectric phase when the strain is less than -0.015 , whereas it is in the ferroelectric phase when the strain is greater than -0.015 .

\section{Concluding remarks}

In summary, a phase field model was employed to study the influence of external applied strains on the spontaneous polarization and the ferroelectric and nonlinear dielectric properties of a single crystal. The simulation results indicate that applied mechanical loads and/or constraints may have the power to tailor the material properties of ferroelectric materials similar to temperature, which allows us to control, modify and fully utilize the material's properties by controlling temperature and/or the mechanical loads and/or constraints.

\section{ACKNOWLEDGEMENTS}

This work was fully supported by an RGC grant from the Research Grants Council of the Hong Kong Special Administrative Region, China. TY Zhang thanks the Croucher Foundation for the Croucher Senior Research Fellowship Award, which gave him more research time by releasing him from his teaching duties. 


\section{APPENDIX}

The elastic energy density equation, Eq. (4), can be rewritten as [28]

$f_{\text {ela }}=\frac{1}{2} c_{11}\left[\left(\varepsilon_{11}^{\text {ela }}\right)^{2}+\left(\varepsilon_{22}^{\text {ela }}\right)^{2}+\left(\varepsilon_{33}^{\text {ela }}\right)^{2}\right]+c_{12}\left[\varepsilon_{11}^{\text {ela }} \varepsilon_{22}^{\text {ela }}+\varepsilon_{22}^{\text {ela }} \varepsilon_{33}^{\text {ela }}+\varepsilon_{11}^{\text {ela }} \varepsilon_{33}^{\text {ela }}\right]+2 c_{44}\left[\left(\varepsilon_{12}^{\text {ela }}\right)^{2}+\left(\varepsilon_{23}^{\text {ela }}\right)^{2}+\left(\varepsilon_{13}^{\text {ela }}\right)^{2}\right]$

with three independent elastic constants, $c_{11}, c_{12}$ and $c_{44}$, according to Voigt's notation. After substituting Eq. (5) and Eq. (11) into Eq. (A1), the elastic energy density can be separated into three parts,

$$
f_{\text {ela }}=f_{\text {ela } 1}+f_{\text {ela } 2}+f_{\text {ela3 }}
$$

in which

$$
\begin{gathered}
f_{\text {ela } 1}=\frac{1}{2} c_{11}\left[\left(\varepsilon_{11}^{(s)}+\varepsilon_{11}^{(a)}\right)^{2}+\left(\varepsilon_{22}^{(s)}+\varepsilon_{22}^{(a)}\right)^{2}+\left(\varepsilon_{33}^{(s)}+\varepsilon_{33}^{(a)}\right)^{2}\right] \\
+c_{12}\left[\left(\varepsilon_{11}^{(s)}+\varepsilon_{11}^{(a)}\right)\left(\varepsilon_{22}^{(s)}+\varepsilon_{22}^{(a)}\right)+\left(\varepsilon_{22}^{(s)}+\varepsilon_{22}^{(a)}\right)\left(\varepsilon_{33}^{(s)}+\varepsilon_{33}^{(a)}\right)\right. \\
\left.+\left(\varepsilon_{11}^{(s)}+\varepsilon_{11}^{(a)}\right)\left(\varepsilon_{33}^{(s)}+\varepsilon_{33}^{(a)}\right)\right] \\
+2 c_{44}\left[\left(\varepsilon_{12}^{(s)}+\varepsilon_{12}^{(a)}\right)^{2}+\left(\varepsilon_{23}^{(s)}+\varepsilon_{23}^{(a)}\right)^{2}+\left(\varepsilon_{13}^{(s)}+\varepsilon_{13}^{(a)}\right)^{2}\right],
\end{gathered}
$$

is the energy density related to the total strain,

$$
f_{\text {ela } 2}=\beta_{11}\left(P_{1}^{4}+P_{2}^{4}+P_{3}^{4}\right)+\beta_{12}\left(P_{1}^{2} P_{2}^{2}+P_{2}^{2} P_{3}^{2}+P_{1}^{2} P_{3}^{2}\right),
$$

is the additional energy density due to the constant strain constraint, and

$$
\begin{aligned}
f_{\text {ela } 3}= & -\left[q_{11}\left(\varepsilon_{11}^{(s)}+\varepsilon_{11}^{(a)}\right)+q_{12}\left(\varepsilon_{22}^{(s)}+\varepsilon_{22}^{(a)}\right)+q_{12}\left(\varepsilon_{33}^{(s)}+\varepsilon_{33}^{(a)}\right)\right] P_{1}^{2} \\
& -\left[q_{11}\left(\varepsilon_{22}^{(s)}+\varepsilon_{22}^{(a)}\right)+q_{12}\left(\varepsilon_{11}^{(s)}+\varepsilon_{11}^{(a)}\right)+q_{12}\left(\varepsilon_{33}^{(s)}+\varepsilon_{33}^{(a)}\right)\right] P_{2}^{2} \\
& -\left[q_{11}\left(\varepsilon_{33}^{(s)}+\varepsilon_{33}^{(a)}\right)+q_{12}\left(\varepsilon_{11}^{(s)}+\varepsilon_{11}^{(a)}\right)+q_{12}\left(\varepsilon_{22}^{(s)}+\varepsilon_{22}^{(a)}\right)\right] P_{3}^{2} \\
- & 2 q_{44}\left[\left(\varepsilon_{12}^{(s)}+\varepsilon_{12}^{(a)}\right) P_{1} P_{2}+\left(\varepsilon_{23}^{(s)}+\varepsilon_{23}^{(a)}\right) P_{2} P_{3}+\left(\varepsilon_{13}^{(s)}+\varepsilon_{13}^{(a)}\right) P_{1} P_{3}\right],
\end{aligned}
$$

is the interaction energy density between total strain and polarization, where

$$
\begin{gathered}
\beta_{11}=\frac{1}{2} c_{11}\left(Q_{11}^{2}+2 Q_{12}^{2}\right)+c_{12} Q_{12}\left(2 Q_{11}+Q_{12}\right), \\
\beta_{12}=c_{11} Q_{12}\left(2 Q_{11}+Q_{12}\right)+c_{12}\left(Q_{11}^{2}+3 Q_{12}^{2}+2 Q_{11} Q_{12}\right)+2 c_{44} Q_{44}^{2},
\end{gathered}
$$




$$
\begin{gathered}
q_{11}=c_{11} Q_{11}+2 c_{12} Q_{12}, \\
q_{12}=c_{11} Q_{12}+c_{12}\left(Q_{11}+Q_{12}\right), \\
q_{44}=2 c_{44} Q_{44} .
\end{gathered}
$$

By combining Eq. (A4) and Eq. (A5) with Eq. (2), the Landau expression can be rewritten as

$$
\begin{aligned}
& f_{L}\left(P_{i}\right)=\alpha_{1}^{e} P_{1}^{2}+\alpha_{2}^{e} P_{2}^{2}+\alpha_{3}^{e} P_{3}^{2} \\
& -2 q_{44}\left[\left(\varepsilon_{12}^{(s)}+\varepsilon_{12}^{(a)}\right) P_{1} P_{2}+\left(\varepsilon_{23}^{(s)}+\varepsilon_{23}^{(a)}\right) P_{2} P_{3}+\left(\varepsilon_{13}^{(s)}+\varepsilon_{13}^{(a)}\right) P_{1} P_{3}\right] \\
& +\left(\alpha_{11}+\beta_{11}\right)\left(P_{1}^{4}+P_{2}^{4}+P_{3}^{4}\right)+\left(\alpha_{12}+\beta_{12}\right)\left(P_{1}^{2} P_{2}^{2}+P_{2}^{2} P_{3}^{2}+P_{1}^{2} P_{3}^{2}\right) \\
& +\alpha_{111}\left(P_{1}^{6}+P_{2}^{6}+P_{3}^{6}\right)+\alpha_{112}\left[\left(P_{1}^{4}\left(P_{2}^{2}+P_{3}^{2}\right)+P_{2}^{4}\left(P_{1}^{2}+P_{3}^{2}\right)+P_{3}^{4}\left(P_{1}^{2}+P_{2}^{2}\right)\right]\right. \\
& +\alpha_{123} P_{1}^{2} P_{2}^{2} P_{3}^{2},
\end{aligned}
$$

in which

$$
\begin{aligned}
& \alpha_{1}^{e}=\alpha_{1}-\left[q_{11}\left(\varepsilon_{11}^{(s)}+\varepsilon_{11}^{(a)}\right)+q_{12}\left(\varepsilon_{22}^{(s)}+\varepsilon_{22}^{(a)}\right)+q_{12}\left(\varepsilon_{33}^{(s)}+\varepsilon_{33}^{(a)}\right)\right], \\
& \alpha_{2}^{e}=\alpha_{1}-\left[q_{11}\left(\varepsilon_{22}^{(s)}+\varepsilon_{22}^{(a)}\right)+q_{12}\left(\varepsilon_{11}^{(s)}+\varepsilon_{11}^{(a)}\right)+q_{12}\left(\varepsilon_{33}^{(s)}+\varepsilon_{33}^{(a)}\right)\right], \\
& \alpha_{3}^{e}=\alpha_{1}-\left[q_{11}\left(\varepsilon_{33}^{(s)}+\varepsilon_{33}^{(a)}\right)+q_{12}\left(\varepsilon_{11}^{(s)}+\varepsilon_{11}^{(a)}\right)+q_{12}\left(\varepsilon_{22}^{(s)}+\varepsilon_{22}^{(a)}\right)\right] .
\end{aligned}
$$

According to the general eigenstrain theory, the local strains, $\varepsilon_{i j}^{(s)}$, are inhomogeneous and their volume averages are zeros, i.e. $\left\langle\varepsilon_{i j}^{(s)}\right\rangle=0$, when the eigenstrains, $\varepsilon_{i j}^{0}$, are periodically distributed in an infinite elastic body [32]. For simplicity, the value of the volume average of Eq. (A8) is usually and approximately used [28]. In this case, Eq. (A8) is reduced to

$$
\begin{aligned}
& \alpha_{1}^{e}=\alpha_{1}-\left(q_{11} \varepsilon_{11}^{(a)}+q_{12} \varepsilon_{22}^{(a)}+q_{12} \varepsilon_{33}^{(a)}\right), \\
& \alpha_{2}^{e}=\alpha_{1}-\left(q_{11} \varepsilon_{22}^{(a)}+q_{12} \varepsilon_{11}^{(a)}+q_{12} \varepsilon_{33}^{(a)}\right), \\
& \alpha_{3}^{e}=\alpha_{1}-\left(q_{11} \varepsilon_{33}^{(a)}+q_{12} \varepsilon_{11}^{(a)}+q_{12} \varepsilon_{22}^{(a)}\right) .
\end{aligned}
$$




\section{References}

[1] Zhang TY, Zhao MH, Tong P. Adv Appl Mech 2002;38:147.

[2] Li Z, Foster M, Dai XH, Xu XZ, Chan SK, Lam DJ. J Appl Phys 1992;71:4481.

[3] Pertsev NA, Zembilgotov AG, Tagantsev AK. Phys Rev Lett 1998;80:1988.

[4] Kwak BS, Erbil A. Phys Rev Lett 1992;68:3733.

[5] Roytburd AL. J. Appl. Phys. 1998;83:228.

[6] Pertsev NA, Koukhar VG. Phys Rev Lett 2000;84:3722.

[7] Oh SH, Jang HM. Appl Phys Lett 1998;72:1457.

[8] Emelyanov AY, Pertsev NA, Kholkin AL. Phys Rev B 2002;66:214108.

[9] Oh SH, Jang HM. Phys Rev B 2000;62:14757.

[10] Amin A, Newnhan RE, Cross LE. Phys Rev B 1986;34:1595.

[11] Koukhar VG, Pertsev NA, Waser R. Appl Phys Lett 2001;78:530.

[12] Kelman MB, Mclntyre PC, Hendrix BC, Bilodeau SM, Roeder JF. J Appl Phys 2003;93:9231.

[13] Oh SH, Jang HM. J Appl Phys 1999;85:2815.

[14] Yamamoto T, Makino Y. Jpn J Appl Phys 1996;35:3214.

[15] Hu HL, Chen LQ. Mater Sci Eng A 1997;238:182.

[16] Hu HL, Chen LQ. J Am Ceram Soc 1998;81:492.

[17] Wang J, Shi SQ, Chen LQ, Li YL, Zhang TY. Acta Mater 2004;52:749.

[18] Ahluwalia R, Lookman T, Saxena A. Phys Rev Lett 2003;91:055501.

[19] Li YL, Choudhury S, Liu ZK, Chen LQ. Appl Phys Lett 2003;83:1608.

[20] Chen LQ, Wolverton C, Vaithyanathan V, Liu ZK. MRS Bulletin, 2001;March issue:197.

[21] Artemev A, Wang Y, Khachaturyan AG. Acta Mater 2000;48:2503. 
[22] Cha PR, Kim SG, Yeon DH, Yoon JK. Acta Mater 2002;50:3817.

[23] Rodney D, Bouar YL, Finel A. Acta Mater 2003;51:17.

[24] Shen C, Wang Y. Acta Mater 2003;51:2595.

[25] Cao W, Cross LE. Phys Rev B 1991;44:5.

[26] Nambu S, Sagala DA. Phys Rev B 1994;50:5838.

[27] Gao YF, Suo Z. J Appl Mech 2002;69:419.

[28] Li YL, Hu SY, Liu ZK, Chen LQ. Acta Mater 2002;50:395.

[29] Hu SY, Li YL, Chen LQ. J Appl Phys 2003;94:2542.

[30] Ahluwalia R, Cao W. J Appl Phys 2001;89:8105.

[31] Ahluwalia R, Cao W. Phys Rev B 2000;63:012103.

[32] Mura T. Micromechanics of defects in solids. Boston: Kluwer Academic Publishers, 1987.

[33] Mitsui T, Tatsuzaki I, Nakamura E. An introduction to the physics of ferroelectrics. London: Gordon and Breach Science Publishers, 1976.

[34] Lines ME, Glass AM. Principles and applications of ferroelectrics and related materials. Oxford : Clarendon press, 1977.

[35] Fatuzzo E, Merz WJ. Ferroelectricity. New York: North-Holland Publishing Co., 1967. 


\section{Figure Captions}

Fig. 1. Domain structures, (a, b, c, d), and the corresponding stress distributions, (A, B, C, D), at $25^{\circ} \mathrm{C}$ under sustained equiaxial strains of 0.008 for (a)-(A); 0 for (b)-(B); -0.008 for (c)-(C); and -0.012 for (d)-(D).

Fig. 2. Temperature dependence of the spontaneous polarization under different sustained equiaxial strains; the inset shows the strain dependence of the phase transition temperature.

Fig. 3. Temperature dependence of the average internal stresses under sustained strains.

Fig. 4. Strain dependence of the average internal stress.

Fig. 5. Temperature dependence of the spontaneous polarization under inequiaxial strains. A equiaxial case is also shown here for comparison.

Fig. 6. Microstructures of ferroelectrics at different temperatures under the applied inequiaxial strains of ${\varepsilon_{11}}^{(a)}=0.004$ and $\varepsilon_{22}{ }^{(a)}=0$.

Fig. 7. Microstructures of ferroelectrics at different temperatures under the applied inequiaxial strains of $\varepsilon_{11}{ }^{(a)}=-0.004$ and $\varepsilon_{22}{ }^{(a)}=0$.

Fig. 8. Microstructures of ferroelectrics at different temperatures under the applied equiaxial strain of $\varepsilon_{11}{ }^{(a)}=\varepsilon_{22}{ }^{(a)}=-0.004$.

Fig. 9. Average polarization versus external applied electric field at different applied equiaxial strains.

Fig. 10. Distribution of polarizations at different applied electric fields with the equiaxial strain of $\varepsilon_{11}{ }^{(a)}=\varepsilon_{22}{ }^{(a)}=-0.02$.

Fig. 11. Distribution of polarizations at different applied electric fields with the equiaxial strain of $\varepsilon_{11}{ }^{(a)}=\varepsilon_{22}{ }^{(a)}=0.01$.

Fig. 12. Coercive electric field and remanent polarization versus applied equiaxial strain.

Fig. 13. Nonlinear dielectric constant versus applied equiaxial strain. 

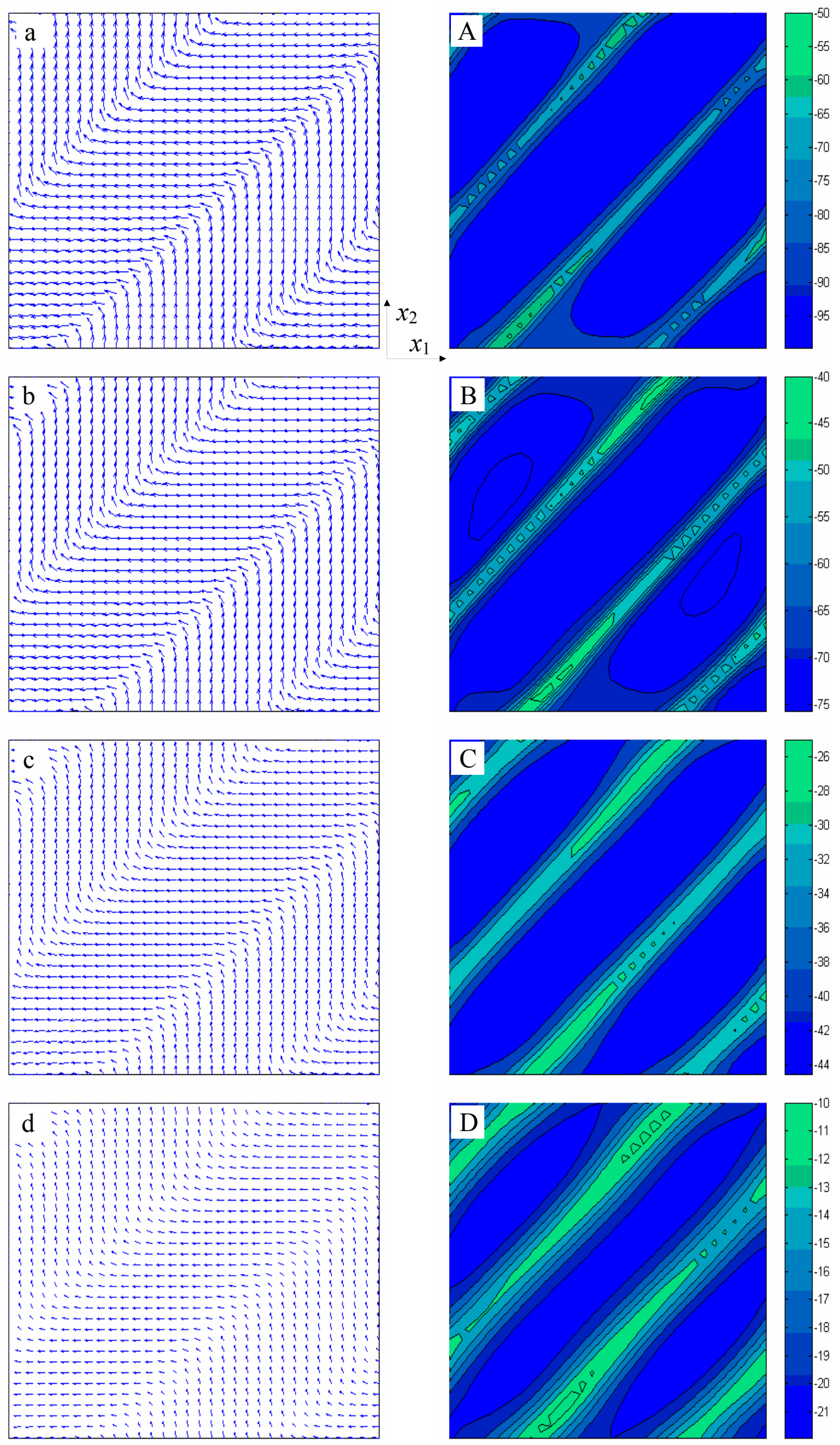

Fig. 1. Domain structures, (a, b, c, d), and the corresponding stress distributions, (A, B, C, D), at $25^{\circ} \mathrm{C}$ under sustained equiaxial strains of 0.008 for (a)-(A); 0 for (b)-(B); -0.008 for (c)-(C); and -0.012 for $(\mathrm{d})-(\mathrm{D})$. 


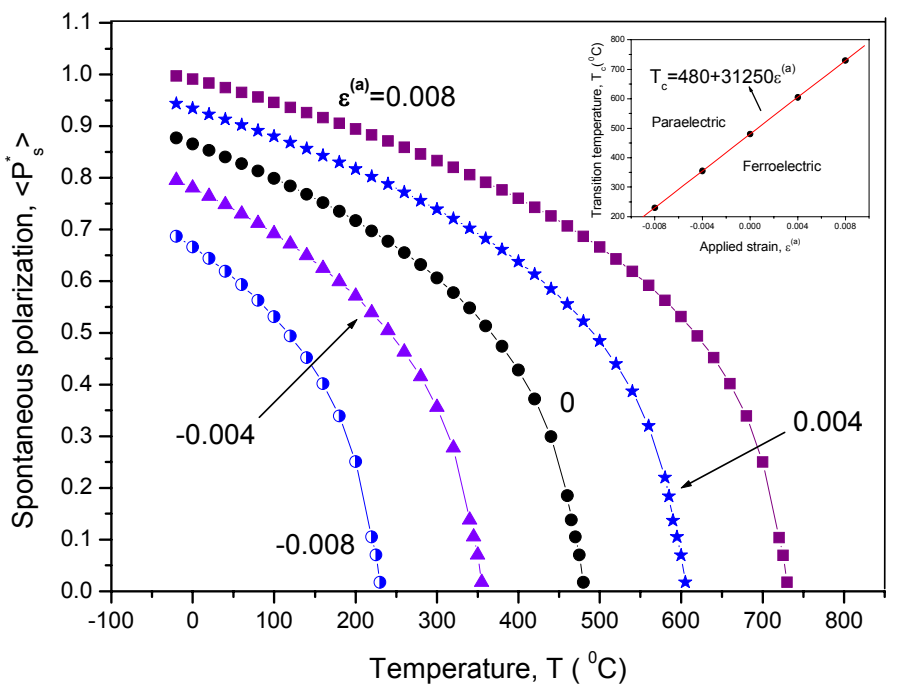

Fig. 2. Temperature dependence of the spontaneous polarization under different sustained equiaxial strains; the inset shows the strain dependence of the phase transition temperature. 


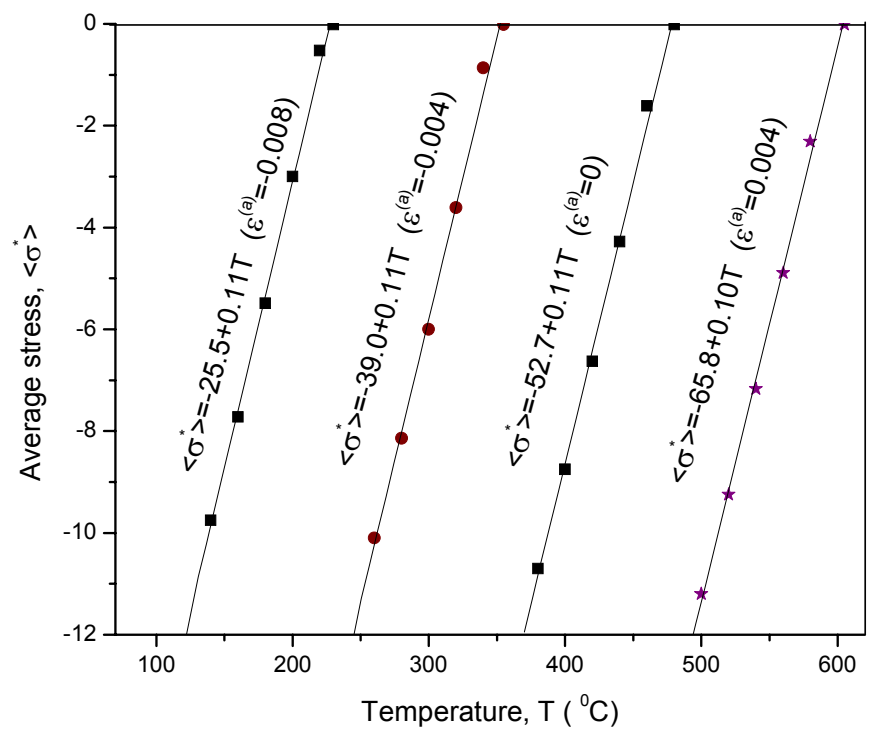

Fig. 3. Temperature dependence of the average internal stresses under sustained strains. 


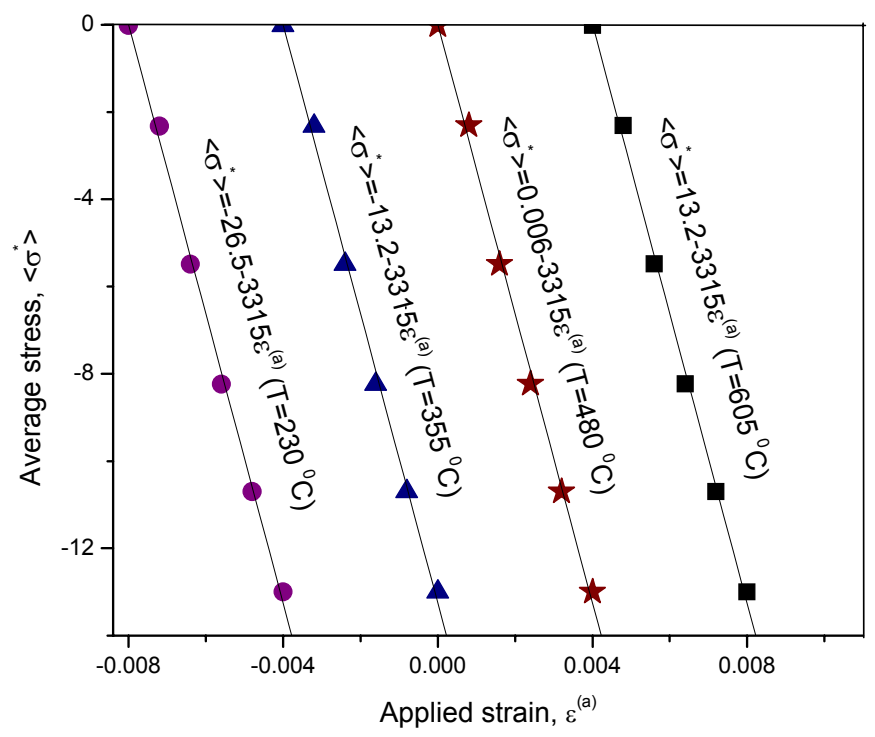

Fig. 4. Strain dependence of the average internal stress. 


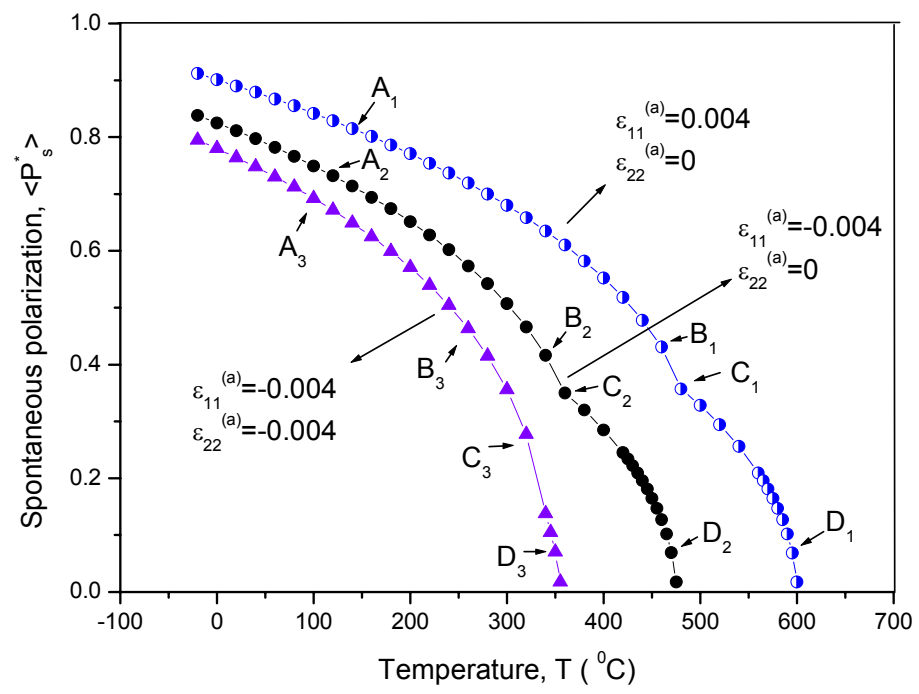

Fig. 5. Temperature dependence of the spontaneous polarization under inequiaxial strains.

A equiaxial case also shown here for comparison. 


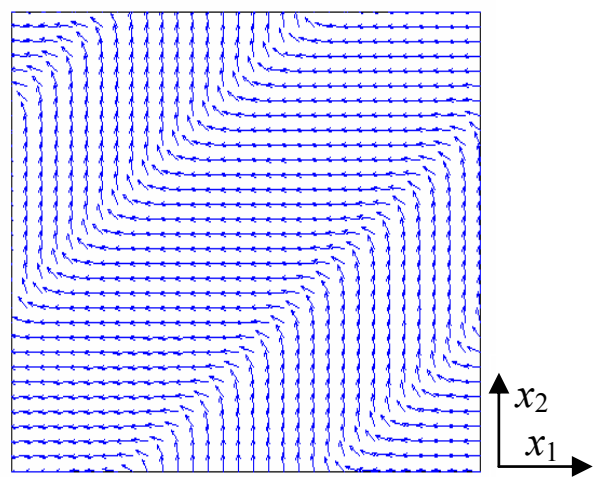

$\left(\mathrm{A}_{1}\right) 140^{\circ} \mathrm{C}$

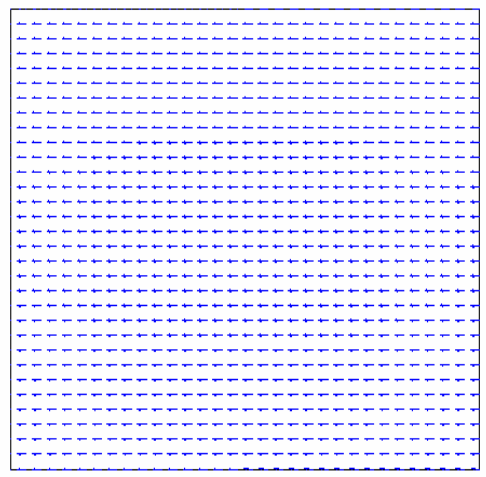

$\left(\mathrm{C}_{1}\right) 480^{\circ} \mathrm{C}$

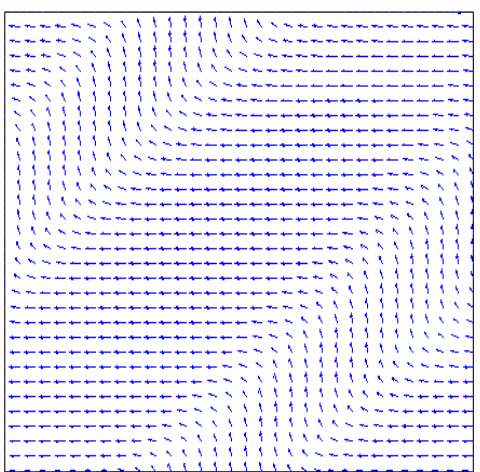

$\left(\mathrm{B}_{1}\right) 460{ }^{\circ} \mathrm{C}$

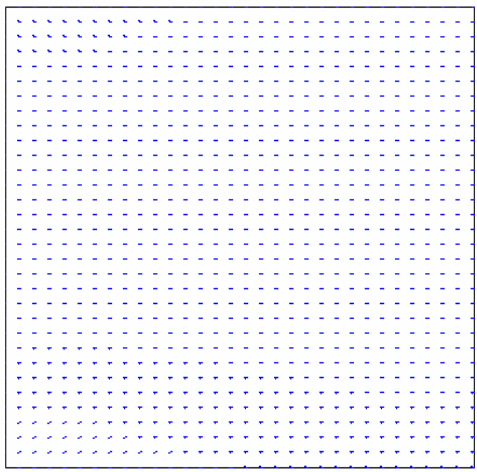

$\left(D_{1}\right) 595^{\circ} \mathrm{C}$

Fig. 6. Microstructures of ferroelectrics at different temperatures under applied inequiaxial strains of $\varepsilon_{11}{ }^{(a)}=0.004$ and $\varepsilon_{22}{ }^{(a)}=0$. 


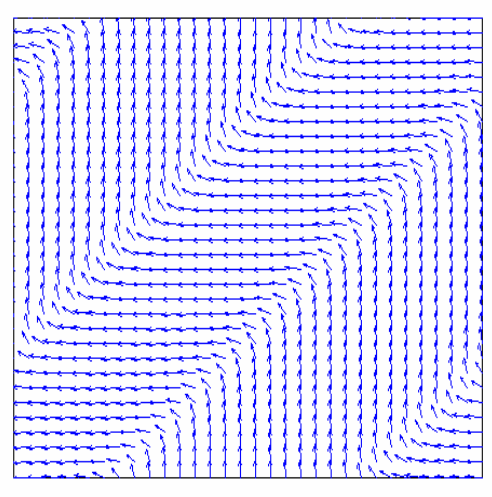

$\left(\mathrm{A}_{2}\right) 120^{\circ} \mathrm{C}$

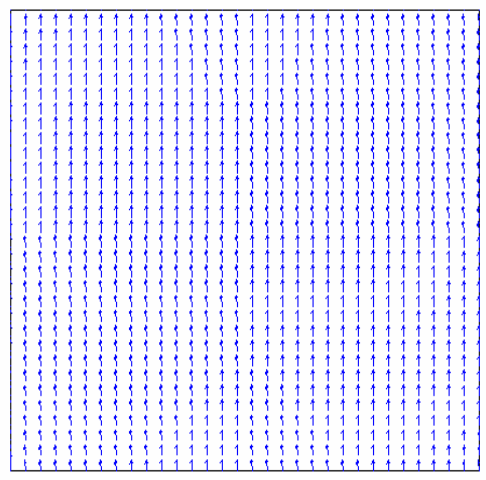

$\left(\mathrm{C}_{2}\right) 360^{\circ} \mathrm{C}$

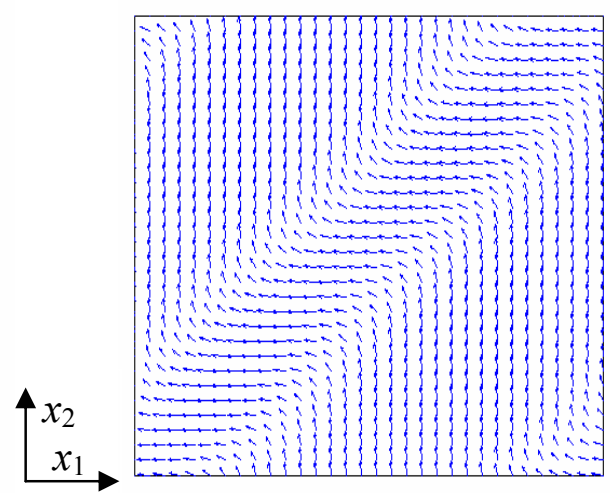

$\left(\mathrm{B}_{2}\right) 340^{\circ} \mathrm{C}$

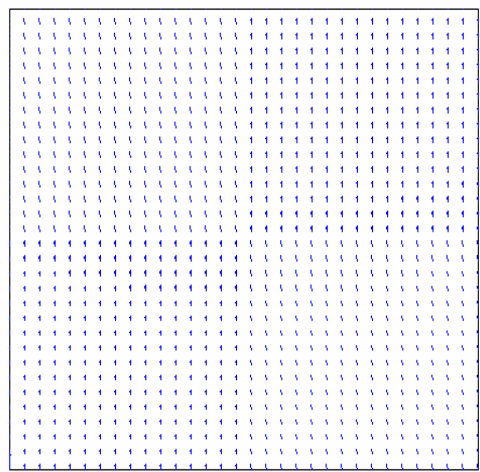

$\left(\mathrm{D}_{2}\right) 470^{\circ} \mathrm{C}$

Fig. 7. Microstructures of ferroelectrics at different temperatures under applied inequiaxial strains of $\varepsilon_{11}{ }^{(a)}=-0.004$ and $\varepsilon_{22}{ }^{(a)}=0$. 


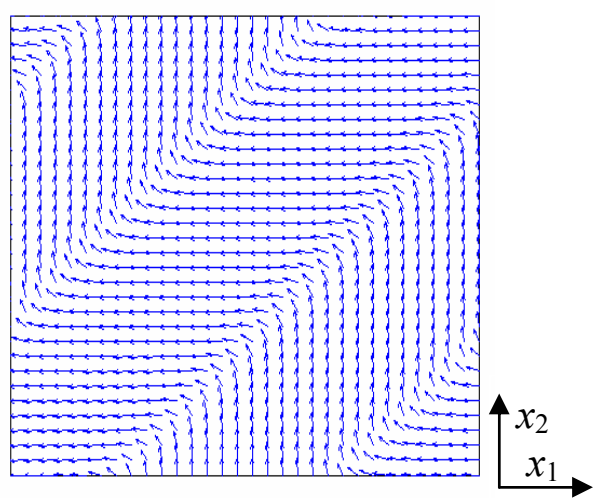

$\left(\mathrm{A}_{3}\right) 100^{\circ} \mathrm{C}$

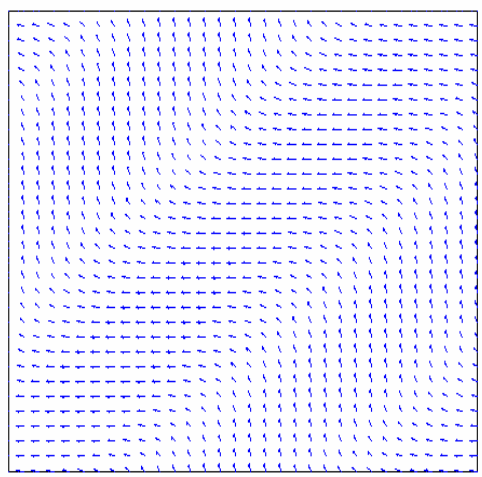

$\left(\mathrm{C}_{3}\right) 320^{\circ} \mathrm{C}$

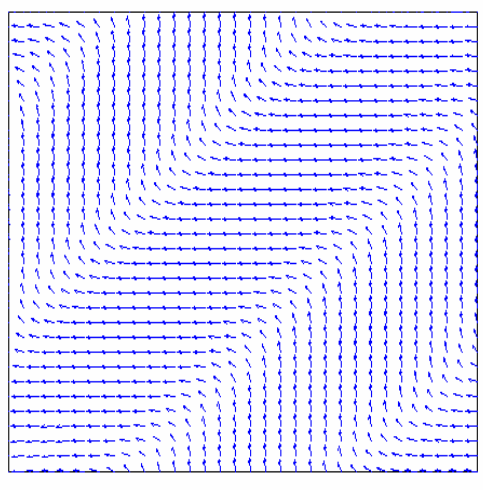

(B 3$) 260^{\circ} \mathrm{C}$

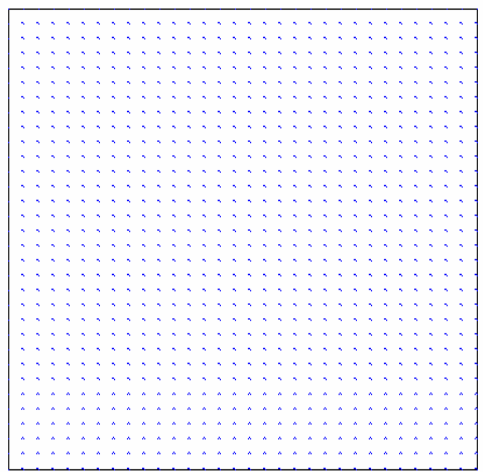

$\left(\mathrm{D}_{3}\right) 350^{\circ} \mathrm{C}$

Fig. 8. Microstructures of ferroelectrics at different temperatures under applied equiaxial strain of $\varepsilon_{11}{ }^{(a)}=\varepsilon_{22}{ }^{(a)}=-0.004$. 


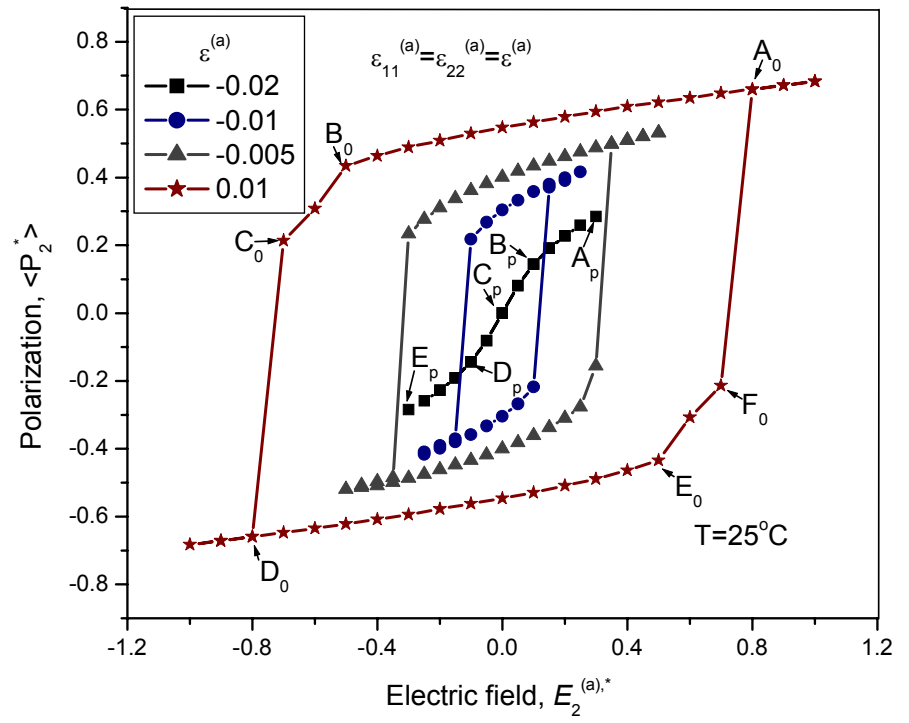

Fig. 9. Average polarization versus external applied electric field at different applied equiaxial strains. 


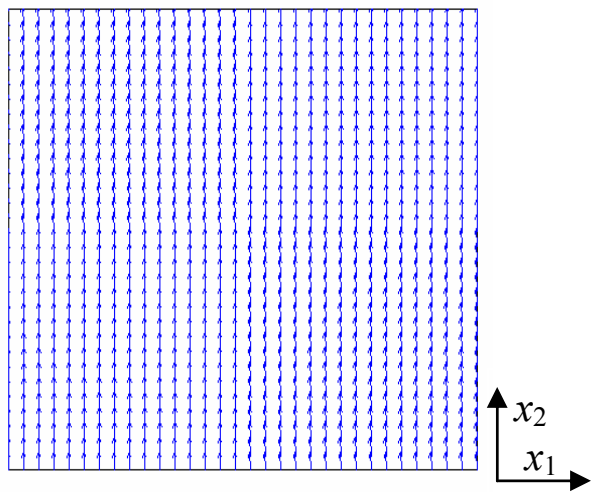

$\left(\mathrm{A}_{\mathrm{p}}\right) E_{2}^{(a),{ }^{*}}=0.35$

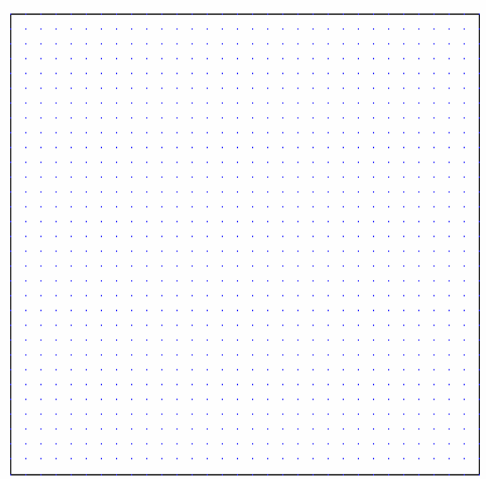

$\left(\mathrm{C}_{\mathrm{p}}\right) E_{2}^{(a), *}=0$

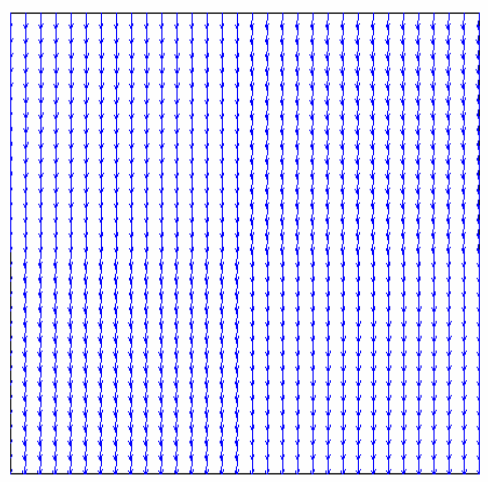

(E $\left.E_{\mathrm{p}}\right) E_{2}^{(a), *}=0.35$

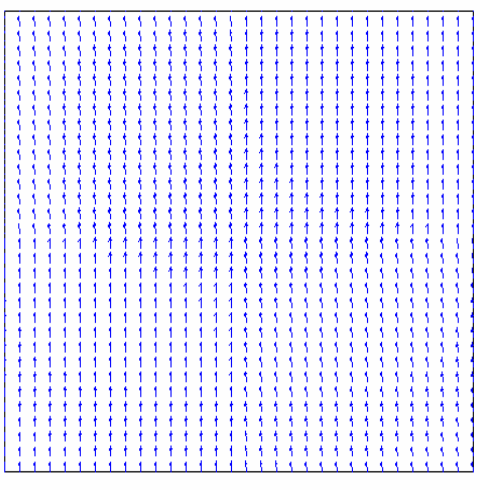

$\left(\mathrm{B}_{\mathrm{p}}\right) E_{2}^{(a),{ }^{*}}=0.1$

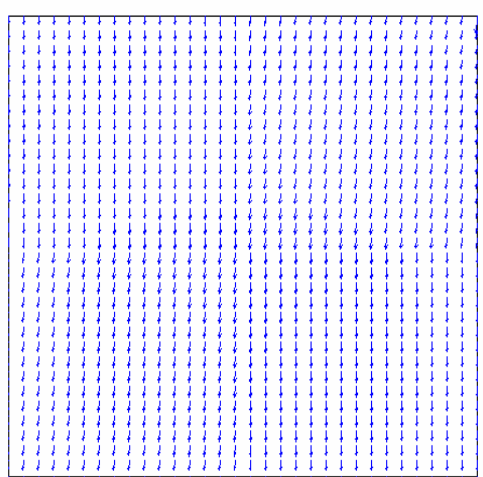

$\left(\mathrm{D}_{\mathrm{p}}\right) E_{2}^{(a), *}=-0.1$

Fig. 10. Distribution of polarizations at different applied electric fields with the equiaxial strain of $\varepsilon_{11}{ }^{(a)}=\varepsilon_{22}{ }^{(a)}=-0.02$. 


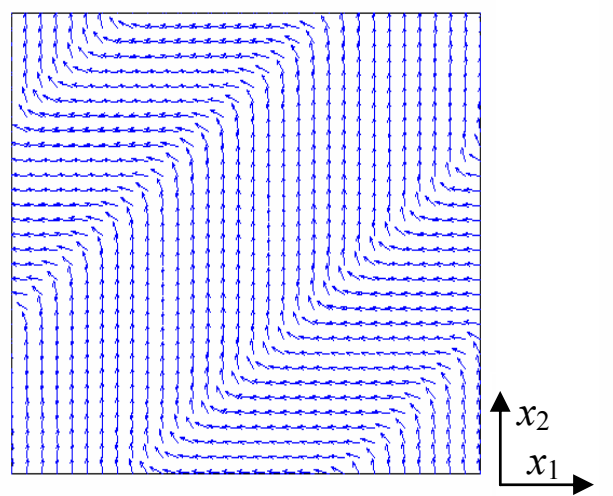

$\left(\mathrm{A}_{0}\right) E_{2}^{(a), *}=0.8$

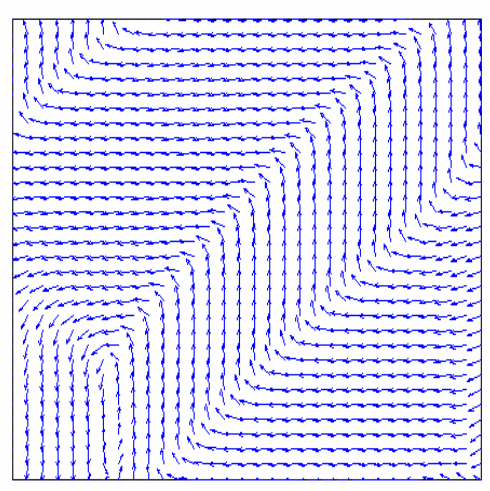

$\left(\mathrm{C}_{0}\right) E_{2}^{(a),{ }^{*}}=-0.7$

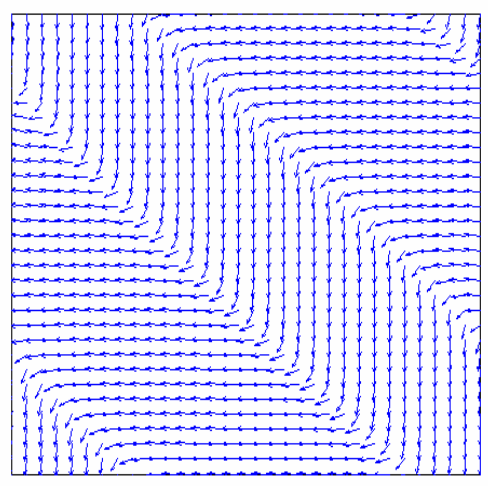

(E) $E_{2}^{(a), *}=0.5$

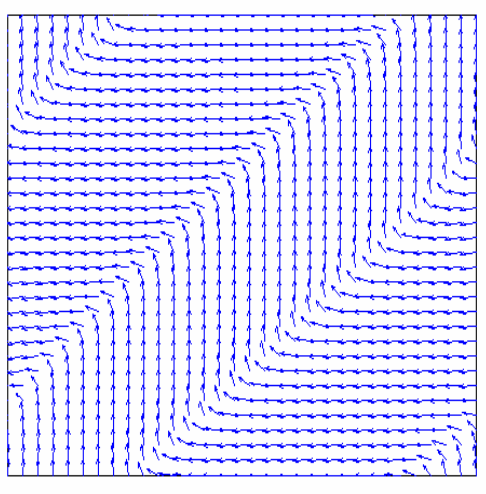

$\left(\mathrm{B}_{0}\right) E_{2}^{(a), *}=-0.5$

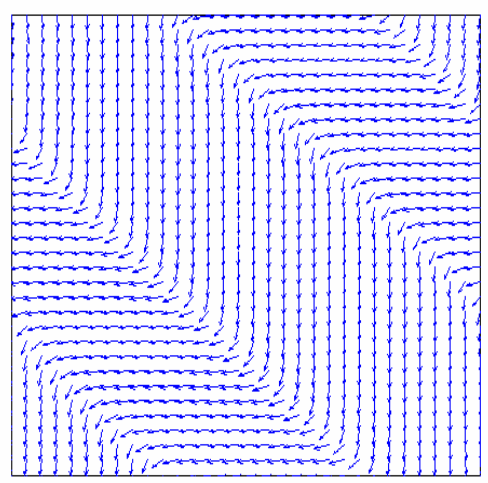

$\left(\mathrm{D}_{0}\right) \quad E_{2}^{(a), *}=-0.8$

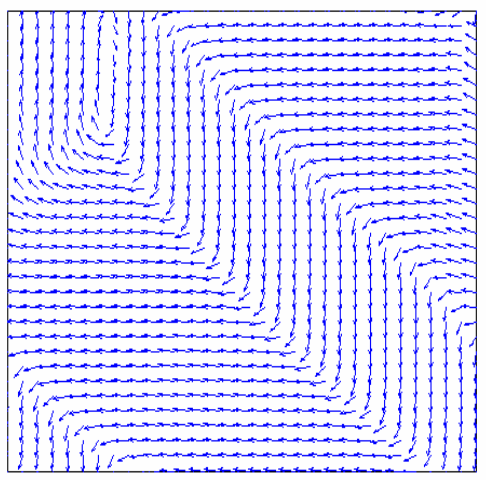

$\left(\mathrm{F}_{0}\right) E_{2}^{(a), *}=0.7$

Fig. 11. Distribution of polarizations at different applied electric fields with the equiaxial strain of $\varepsilon_{11}{ }^{(a)}=\varepsilon_{22}{ }^{(a)}=0.01$. 


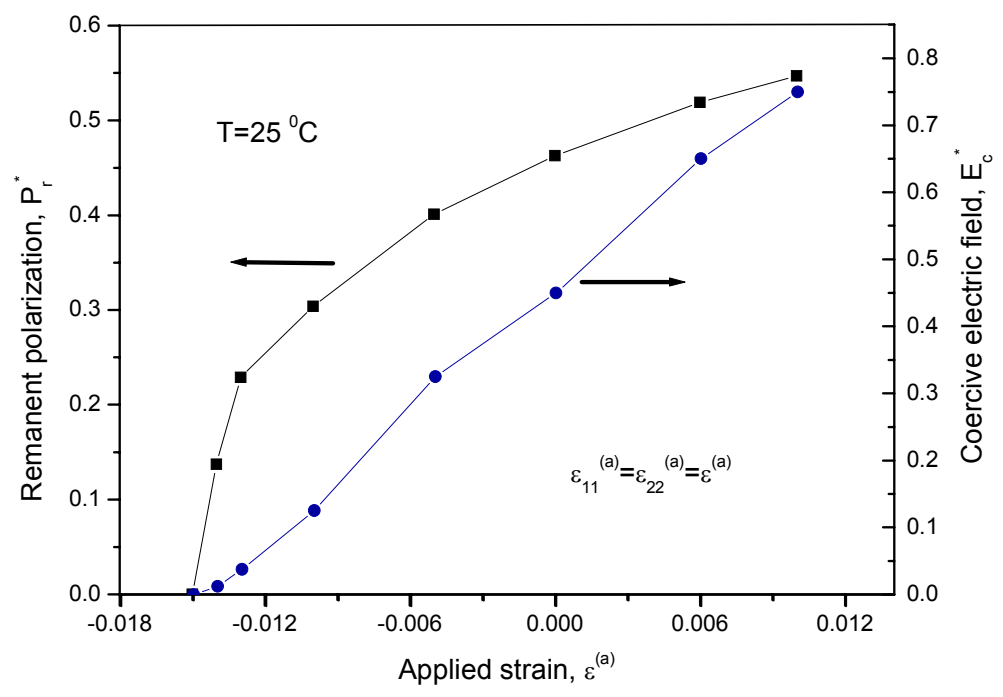

Fig. 12. Coercive electric field and remanent polarization versus applied equiaxial strain. 


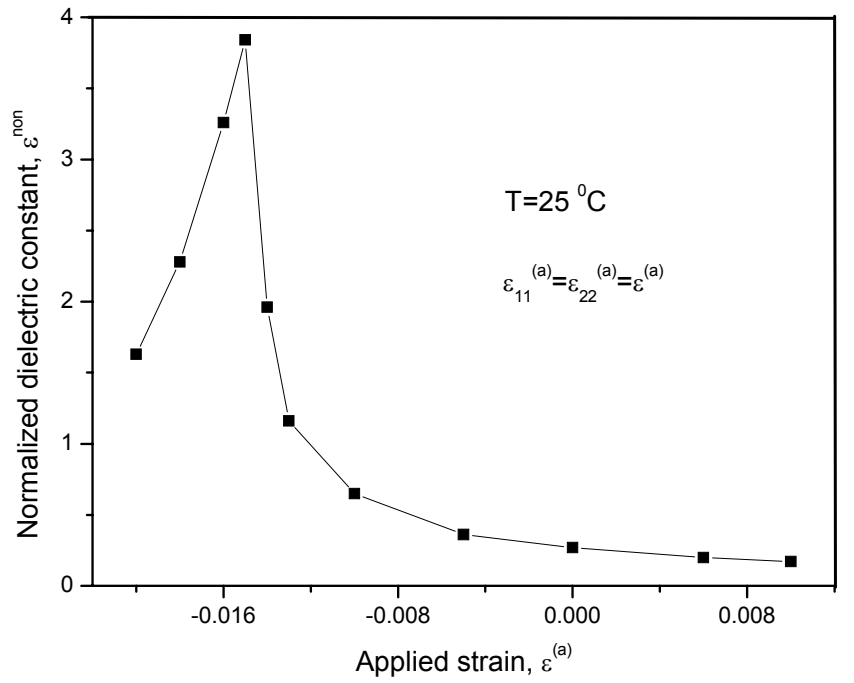

Fig. 13. Nonlinear dielectric constant versus applied equiaxial strain. 